\title{
Abordaje endoscópico del seno frontal mediante Draf III. Experiencia de la Red de Salud UC Christus
}

\section{The Draf III procedure as an endoscopic frontal sinus approach. Red de Salud UC Christus' experience}

\author{
Sofia Waissbluth A', Tamara Muñoz M¹, Ximena Maul F¹, Claudia González G1', Claudio Callejas C.
}

\section{RESUMEN}

Introducción: El Draf III es una técnica quirúrgica que permite el abordaje endoscópico de los senos frontales. Fue descrita como un procedimiento de rescate para la rinosinusitis crónica (RSCr) frontal en que falla la cirugía estándar. Actualmente, su uso se ha extendido a otras aplicaciones.

Objetivo: (1) Revisar las indicaciones del abordaje Draf III, (2) Revisar si el abordaje permitió el manejo adecuado de la patología y (3) Evaluar la permeabilidad de los senos frontales.

Material y método: Estudio retrospectivo descriptivo. Se evaluaron los registros de pacientes sometidos a un abordaje Draf III entre los años 2013-2016 $(n=11)$. Se revisaron las indicaciones quirúrgicas, utilidad del Draf III para manejar la patología, permeabilidad de los senos frontales y complicaciones quirúrgicas.

Resultados: Indicaciones quirúrgicas incluyeron la RSCr con pólipos, osteoma frontoetmoidal, mucocele frontal, papiloma invertido y estesioneuroblastoma. En todos los casos, el abordaje permitió un adecuado manejo de la patología. Todos los pacientes presentaron permeabilidad adecuada del Draf III al último control. Un paciente debió ser reoperado a los 15,5 meses por recidiva de papiloma invertido. Ningún paciente requirió cirugía de revisión por estenosis crítica del Draf III. No hubo complicaciones quirúrgicas.

Discusión: El Draf III fue útil para manejar la RSCr, patologías benignas del seno frontal, y como parte de un abordaje extendido a la fosa craneal anterior.

Conclusión: En nuestra serie, el Draf III resultó ser una excelente opción quirúrgica para el tratamiento de diversas patologías del seno frontal.

Palabras clave: Draf III, procedimiento de Lothrop endoscópico modificado, seno frontal, cirugía endoscópica nasal.

\section{ABSTRACT}

Introduction: The Draf III procedure is an effective approach for the management of frontal sinus disorders. It was originally described as a rescue procedure for the treatment of refractory frontal sinusitis. Currently, it can be used to treat a variety of other disorders.

\footnotetext{
1 Departamento Otorrinolaringología, Pontificia Universidad Católica de Chile, Santiago, Chile.

*Los autores declaran no tener conflictos de interés.

Recibido el 31 de mayo, 2018. Aceptado el 14 de agosto, 2018.
} 
Aim: (1) To review the indications for the Draf III procedure, (2) To evaluate if it enabled appropriate management of the disease, and (3) To evaluate frontal sinus patency.

Material and Method: Retrospective descriptive study. Clinical records of patients who underwent a Draf III procedure between the years 2013-2016 (n=11) were reviewed. Surgical indications, the appropriateness of the approach to manage the frontal sinus disease, frontal sinus patency, and complications were analyzed.

Results: Surgical indications included chronic rhinosinusitis with polyps, frontoethmoidal osteoma, frontal mucocele, inverted papilloma and esthesioneuroblastoma. In all cases, the approach allowed adequate management of the pathology. All patients had adequate patency of the Draf III at their last follow-up. One patient required a revision Draf III at 15.5 months follow-up because of an inverted papilloma recurrence. No patient required revision surgery for critical stenosis of the Draf III. No surgical complications were observed.

Discussion: The Draf III procedure was useful for the management of chronic rhinosinusitis, for benign frontal sinus pathologies, and as part of an extended approach to the anterior cranial fossa.

Conclusion: In our series, the Draf III procedure was an excellent surgical option for the management of a variety of frontal sinus pathologies.

Key words: Draf III, modified endoscopic Lothrop procedure, frontal sinus, endoscopic sinus surgery.

\section{INTRODUCCIÓN}

El abordaje de patologías de los senos frontales puede ser un desafío para el otorrinolaringólogo. Varias opciones quirúrgicas han sido descritas incluyendo abordajes externos, intranasales o combinados. Con el desarrollo de la cirugía endoscópica nasal, los abordajes endoscópicos se han ido adoptando cada vez más, principalmente debido a su menor morbilidad asociada y excelente resultados.

El procedimiento Draf $\mathrm{III}^{1}$, o procedimiento de Lothrop endoscópico modificado, es un abordaje inicialmente descrito como un procedimiento endoscópico de rescate ante el fracaso de la cirugía endoscópica funcional en el manejo de la rinosinusitis crónica (RSCr) del seno frontal. Debido a la menor morbilidad asociada y excelentes resultados, desplazó al flap osteoplástico con obliteración con grasa, considerado por muchos años como el estándar de oro para el manejo del seno frontal cicatricial2.

El procedimiento consiste en crear la mayor comunicación posible entre ambos senos frontales y las fosas nasales. Para esto es necesario remover el piso de ambos senos frontales, el tabique interseno frontal y una porción del tabique nasal bajo los senos frontales para crear una ventana septal que permita trabajar desde una fosa nasal hacia la otra. Esto implica fresar el proceso frontal de los huesos maxilares y la espina nasal del frontal. Los límites de la disección son el periostio bajo la piel de la región del nasión anterior y lateralmente, y la primera neurona olfatoria hacia posterior ${ }^{1}$.

Con el desarrollo de la cirugía endoscópica nasal, las aplicaciones del Draf III se han ampliado. Actualmente incluyen la resección de diversos tipos de tumores del seno frontal, cierre de fístulas de líquido cefalorraquídeo, manejo de fracturas del seno frontal y como parte de abordajes endonasales extendidos a la fosa craneal anterior, entre otras $^{3}$.

\section{OBJETIVO}

(1) Revisar las indicaciones del abordaje Draf III en la Red de Salud UC Christus, (2) revisar si el abordaje permitió el manejo adecuado de la patología del seno frontal en cada caso y, (3) evaluar la permeabilidad de los senos frontales en el posoperatorio a corto, mediano y largo plazo.

\section{MATERIAL Y MÉTODO}

Se realizó un estudio retrospectivo, descriptivo, de todos los pacientes sometidos a un abordaje 
tipo Draf III manejados por el equipo de cirugía endoscópica de cavidades paranasales de la Red de Salud UC Christus, entre los años 2013 y 2016, en el Hospital Clínico UC y Clínica San Carlos de Apoquindo. El estudio cuenta con la aprobación del Comité de Ética de la Facultad de Medicina de la Pontificia Universidad Católica de Chile. Todas las cirugías fueron realizadas por el autor senior del trabajo (C.C.C.).

Se revisaron las bases de datos (fichas electrónicas, imágenes y protocolos operatorios) para obtener la siguiente información: 1) indicación quirúrgica, 2) determinar si el abordaje permitió el manejo adecuado de la patología del seno frontal en cada caso, 3) permeabilidad de los senos frontales a corto (menor a 12 meses), mediano (entre 12 y 24 meses) y largo plazo (mayor a 24 meses), y 4) complicaciones quirúrgicas. Se realizó seguimiento clínico de la permeabilidad de los senos frontales con endoscopía nasal hasta enero de 2018. La permeabilidad se clasificó como adecuada si la abertura lograda permitía el adecuado drenaje de los senos frontales. La permeabilidad adecuada se subdividió en: 1) amplia, si ambos senos frontales estaban ampliamente expuestos y fácilmente controlables con endoscopio de 0 grados y 2) parcial, si existían sinequias que obstruyeran parcialmente la visualización al interior de algún seno frontal, pero no impedían el drenaje de éstos. La permeabilidad se clasificaría como 3) inadecuada, si se desarrollaba una estenosis crítica del Draf III que no permitiera el drenaje adecuado de los senos y por lo tanto requiriera una cirugía de revisión para repermeabilizar el Draf III. Las complicaciones quirúrgicas evaluadas incluyeron el sangrado posoperatorio, fístulas de líquido cefalorraquídeo, alteraciones oculares, alteraciones olfatorias y el empeoramiento de los síntomas. Se incluyeron 11 pacientes en total, 6 hombres y 5 mujeres. La edad promedio fue de $51,4 \pm 12,3$ años (rango 30-67 años).

\section{RESULTADOS}

\section{Indicaciones quirúrgicas}

La Tabla 1 resume las indicaciones de Draf III en los 11 pacientes del estudio. La indicación principal estuvo relacionada a $\mathrm{RSCr}$, debido a fracaso de tratamiento previo con cirugía endoscópica estándar (Figura 1A).

En un caso, se decidió realizar un Draf III primario (paciente con antecedente de antrostomías maxilares, pero sin antecedente de cirugía de los recesos frontales previa) debido a la compleja anatomía del receso frontal que presentaba el paciente (Figura 1B).

\section{Utilidad del abordaje Draf III para el manejo de la patología frontal}

En todos los casos, el Draf III permitió un adecuado acceso a los senos frontales para manejar en forma endoscópica la patología que los afectaba. En los casos de RSCr, permitió un adecuado acceso para limpiar el contenido en senos frontales (pólipos y moco eosinofílico). Además, la gran abertura lograda permitió mantener la permeabilidad de los senos frontales cicatriciales en todos los casos. En el caso con anatomía compleja, permitió resecar todas las celdillas que se extendían en forma significativa al interior de los senos frontales y los pólipos asociados. En los casos de osteoma, permitió adecuado acceso para resecarlos. En ambos casos, los pacientes presentaban además RSCr con pólipos con indicación quirúrgica debido a fracaso a terapia médica máxima, la cual se resolvió quirúrgicamente en el mismo acto. Uno de los casos se trataba de un osteoma gigante con gran extensión a la órbita ${ }^{4}$ (Figura 2).

En este caso, el abordaje Draf III permitió una adecuada exposición para fresar su interior y luego fracturar desde el exterior su "cáscara" hacia la fosa nasal para completar la resección. Esta paciente recuperó completamente su olfato tras presentar anosmia por años. En los casos de mucocele (Figura 3), se intentó inicialmente un abordaje endoscópico estándar del receso frontal.

Sin embargo, dada a la imposibilidad de realizar una disección adecuada debido a la existencia de neosteogénesis en la zona del receso frontal, fue necesario realizar el Draf III que permitió una amplia marsupialización de los mucoceles y mantener la permeabilidad de la abertura en el tiempo. En los casos de papiloma invertido, permitió un adecuado acceso para resecar el tumor y fresar el sitio de implantación. Si bien una paciente recidivó 
Tabla 1. Resumen de los pacientes sometidos a un abordaje tipo Draf III ordenados por diagnóstico

\begin{tabular}{|c|c|c|c|c|c|c|}
\hline Paciente & Edad & Sexo & Diagnósticos & $\begin{array}{l}\text { CEN }^{\mathrm{a}} \\
\text { previa }\end{array}$ & $\begin{array}{l}\text { Permeabilidad Draf III } \\
\text { al último control }\end{array}$ & $\begin{array}{l}\text { Tiempo de } \\
\text { seguimiento }\end{array}$ \\
\hline 1 & 33 & M & Rinosinusitis crónica con pólipos & $\mathrm{Si}(1 \mathrm{x})$ & Ampliamente permeable & 30 meses \\
\hline 2 & 61 & $\mathrm{~F}$ & Rinosinusitis crónica con pólipos - ADM ${ }^{b}$ & $\mathrm{Si}(1 \mathrm{x})$ & $\begin{array}{l}\text { Ampliamente permeable, } \\
\text { edema de mucosa zona Draf III }\end{array}$ & 34 meses \\
\hline 3 & 55 & M & Rinosinusitis crónica con pólipos - Asma & $\mathrm{Si}(8 \mathrm{x})$ & Ampliamente permeable & 19 meses \\
\hline 4 & 57 & $\mathrm{~F}$ & $\begin{array}{l}\text { Rinosinusitis crónica con pólipos - } \\
\text { Tríada de Samter }\end{array}$ & Si $(4 \mathrm{x})$ & $\begin{array}{l}\text { Parcial, pero adecuado. } \\
\text { Edema de mucosa }\end{array}$ & 19 meses \\
\hline 5 & 60 & $\mathrm{~F}$ & $\begin{array}{l}\text { Osteoma frontoetmoidal } \\
\text { Rinosinusitis crónica con pólipos }\end{array}$ & No & Ampliamente permeable & 34 meses \\
\hline 6 & 67 & M & $\begin{array}{l}\text { Osteoma frontoetmoidal } \\
\text { Rinosinusitis crónica con pólipos }\end{array}$ & No & Ampliamente permeable & 10 meses \\
\hline 7 & 41 & M & Mucocele frontal & No & Parcial, pero suficiente & 23 meses \\
\hline 8 & 61 & M & Mucocele frontoetmoidal & No & Ampliamente permeable & 23 meses \\
\hline 9 & 30 & $\mathrm{~F}$ & Papiloma invertido frontal & $\begin{array}{l}\text { No } \rightarrow \\
\text { Draf III }\end{array}$ & $\begin{array}{l}\text { Ampliamente } \rightarrow \\
\text { Parcial, suficiente } \rightarrow\end{array}$ & $\begin{array}{l}15.5 \text { meses }^{*} \\
5 \text { meses } ¥\end{array}$ \\
\hline 10 & 45 & M & Papiloma invertido frontal & No & Parcial, pero adecuado & 4 meses \\
\hline 11 & 55 & $\mathrm{~F}$ & Estesioneuroblastoma (Kadish C) & No & Ampliamente permeable & 24 meses \\
\hline
\end{tabular}

a $\quad$ CEN: cirugía endoscópica nasal.

b $\quad$ ADM: asma difícil manejo. Requirió omalizumab.

*: $\quad$ Primera cirugía.

¥: $\quad$ Cirugía de revisión del Draf III.

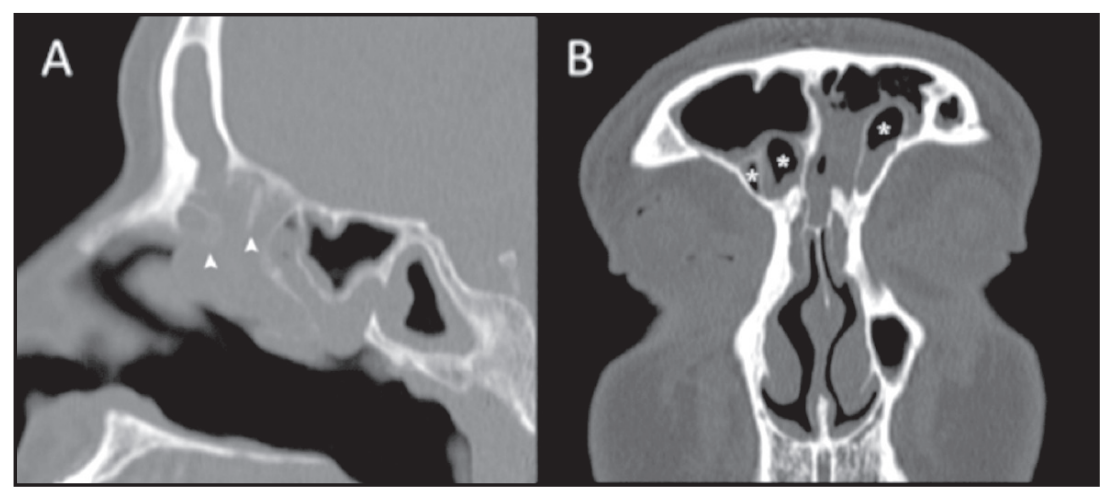

Figura 1. Tomografía computarizada que ejemplifica casos de rinosinusitis crónica con pólipos operados con abordaje Draf III. (A) Corte sagital: paciente previamente operada. Se intentó realizar una cirugía estándar, pero por la presencia de cicatrices en los recesos frontales, neosteogénesis, y dificultad en identificar los reparos anatómicos, se decide realizar un Draf III (Paciente \#2). Nótese la cirugía parcial del receso frontal con celdillas y tabiques remanentes, que posteriormente evolucionaron en sinequias y neosteogénesis (cabezas de flecha). (B) Corte coronal: caso con anatomía frontal compleja con celdillas supraagger frontales extensas bilaterales (Paciente \#1) ${ }^{\star}$, celdillas supraagger frontales. 


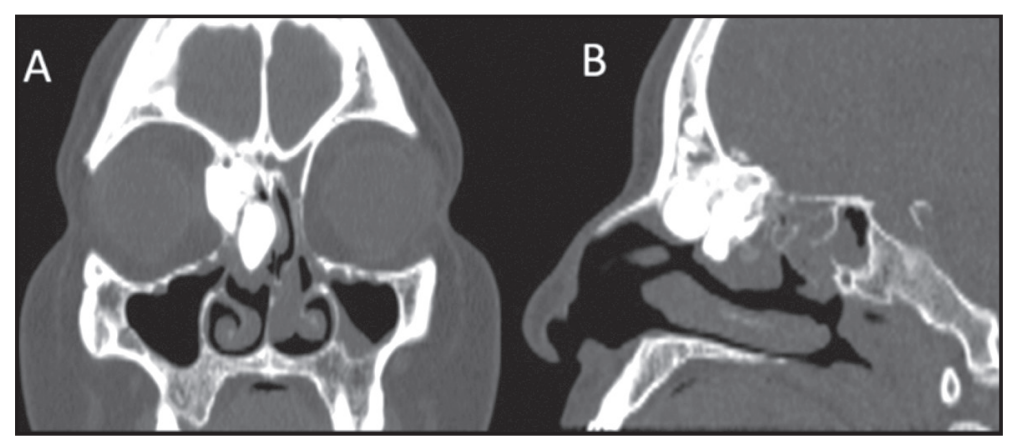

Figura 2. Tomografía computarizada de un osteoma frontoetmoidal derecho con extensión orbitaria (Paciente \#5). (A) Corte coronal y (B) sagital.

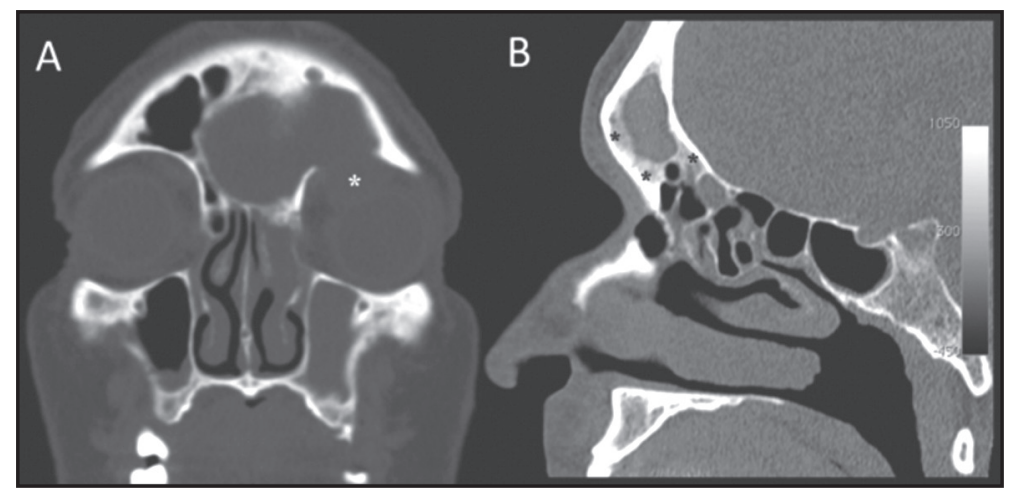

Figura 3. Tomografía computarizada de un mucocele frontal operado mediante Draf III (Paciente \#7). (A) Corte coronal: mucocele frontal izquierdo que se extiende a órbita ipsilateral ${ }^{\star}$, extensión orbitaria de mucocele. (B) Corte sagital: se observa neosteogénesis en el seno y receso frontal ${ }^{\star}$, neosteogénesis.

a los 15,5 meses (Figura 4, paciente \#9 en Tabla 1), creemos que no se debió a un inadecuado acceso quirúrgico, sino a que en la primera cirugía no se fresó parte del sitio de inserción del papiloma invertido en la lamela lateral de la lámina cribosa (pese a tener un acceso cómodo para realizarlo) debido al riesgo de crear una fístula de líquido cefalorraquídeo. En la segunda cirugía, se decidió fresar en forma cuidadosa este sitio. Al momento de su último control posoperatorio la paciente se mantenía libre de recidiva. La paciente se perdió de los controles a los 5 meses de seguimiento de su cirugía de revisión.

Por último, en el abordaje endoscópico endonasal extendido a la fosa craneal anterior para resección de un estesioneuroblastoma Kadish $C^{5}$
(Figura 5), el Draf III fue necesario para resecar la lámina cribosa hasta su extensión más anterior incluyendo la crista galli. Esto debido a la extensión anterior del tumor más allá de las arterias etmoidales anteriores.

\section{Permeabilidad}

Todos los pacientes $(n=11)$ presentaron permeabilidad adecuada del Draf III al último control. El promedio de seguimiento fue de 20,3 meses (rango: 4-34 meses). Ningún paciente requirió cirugía de revisión debido a estenosis crítica del Draf III. Cuatro pacientes presentaron más de 24 meses de seguimiento (30 meses de seguimiento promedio) y persistieron con una abertura de 


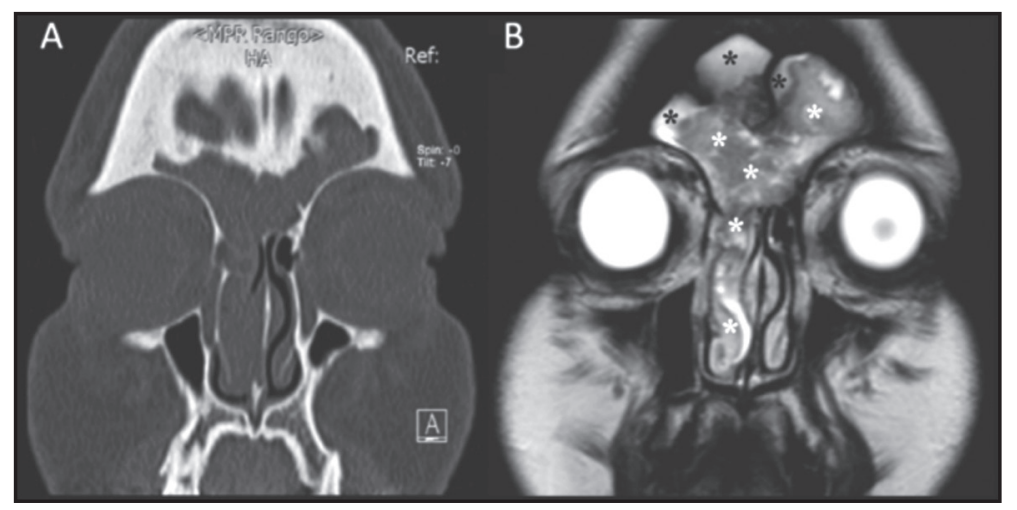

Figura 4. Imágenes de papiloma invertido frontal. (A) Tomografía computarizada, corte coronal. (B) Resonancia magnética (RM) de cavidades perinasales (imagen potenciada en T2), corte coronal. Nótese como la RM permite diferenciar secreciones retenidas de tumor. En este caso se observa extenso compromiso de ambos senos frontales (Paciente \#9). Asteriscos negros, secreciones. Asteriscos blancos, tumor.

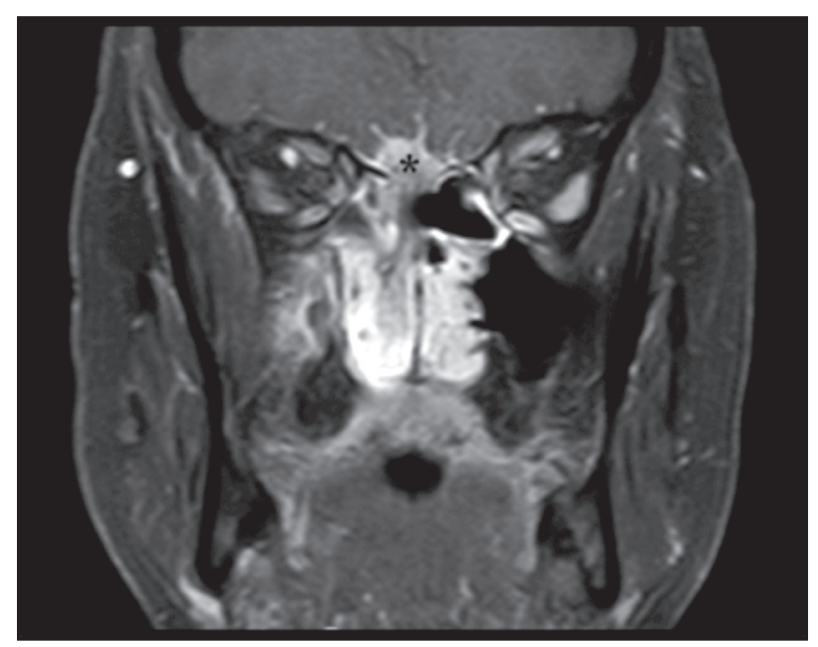

Figura 5. Resonancia magnética de cavidades paranasales potenciada en T1 con gadolinio, corte coronal. Se observa una lesión de partes blandas polipoidea ocupando la fosa nasal derecha. Se acompaña de un engrosamiento leptomeníngeo anormal que compromete los surcos olfatorios y surcos frontales basales, así como el aspecto más inferior de la cisura interhemisférica (Paciente \#11)*, extensión endocraneal del tumor.

Ios senos frontales amplia y estable en el tiempo (Figura6A). Cuatro pacientes tuvieron seguimiento entre 12 y 24 meses y presentaron permeabilidad adecuada (seguimiento promedio de 21 meses). Tres de éstos presentaron permeabilidad amplia y uno permeabilidad parcial, pero suficiente (Figura 6B).

Tres pacientes presentaron un periodo de seguimiento menor a 12 meses $(6,3$ meses de seguimiento promedio). Una paciente operada de papiloma invertido (paciente \#9 en Tabla 1), inicialmente presentó una permeabilidad amplia hasta los 15,5 meses de seguimiento. Sin embargo, requirió ser reoperada por recidiva de papiloma invertido y presentó luego una permeabilidad parcial, pero suficiente, al momento de su último control a los 5 meses de la cirugía de revisión. Por lo anterior fue incluida en el grupo de pacientes 


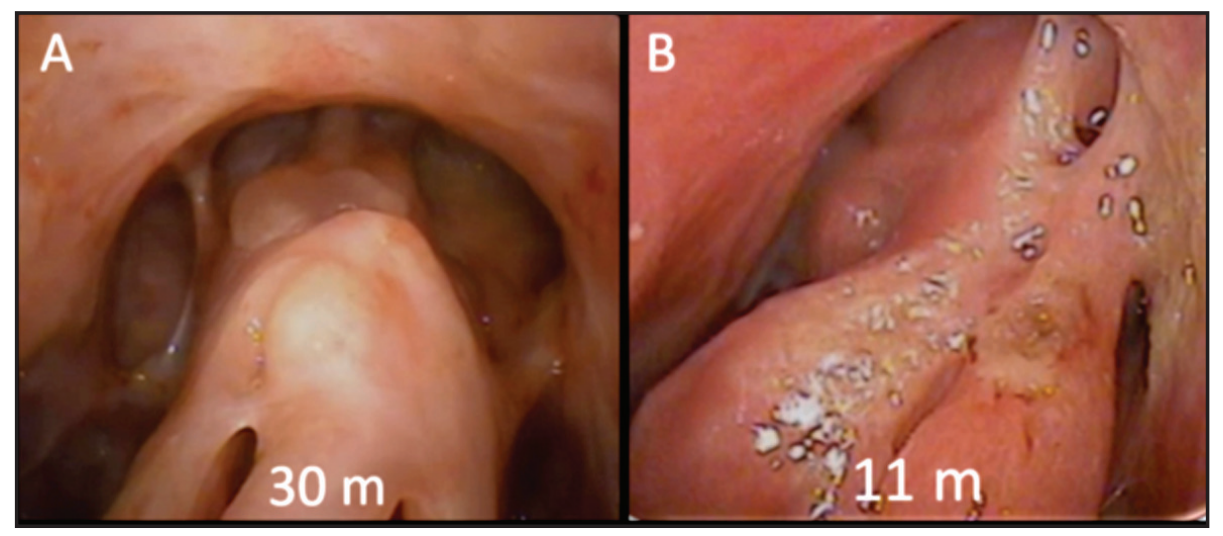

Figura 6. Visualización de los senos frontales por vía endoscópica. (A) Caso con seguimiento a 30 meses, se observa permeabilidad amplia de los senos frontales (Paciente \#1). (B) Caso con seguimiento de 23 meses; endoscopía realizada a los 11 meses, que representa el aspecto hasta el final del estudio, con permeabilidad parcial pero suficiente (Paciente \#7).

con menos de 12 meses de seguimiento para el análisis. Otro paciente se perdió de seguimiento a los 4 meses porque se mudó a vivir al extranjero (permeabilidad parcial, pero adecuada). El tercer paciente llevaba 10 meses de seguimiento en su último control (permeabilidad amplia).

\section{Complicaciones}

No hubo complicaciones quirúrgicas como sangrado posoperatorio, fistula de líquido cefalorraquídeo, alteraciones oculares ni olfatorias. Ningún paciente presentó empeoramiento de sus síntomas.

\section{DISCUSIÓN}

En nuestra experiencia, el Draf III ha resultado ser un excelente abordaje para manejar una variada gama de patologías que afectan al seno frontal. Con el desarrollo de la cirugía endoscópica nasal, el Draf III ha adquirido una gran popularidad para manejar una múltiple variedad de patologías que afectan a los senos frontales, desplazando a los abordajes abiertos en gran parte debido a su menor morbilidad asociada, menor estadía hospitalaria y excelentes resultados. Pese a lo anterior, el abordaje bicoronal o flap osteoplástico sigue teniendo un rol importante para manejar patología del seno frontal localizada en áreas de difícil acceso mediante un Draf III, como la periferia de senos frontales con neumatización importante.

En relación a las indicaciones de Draf III en nuestra serie, la más frecuente fue RSCr, debido a fracaso de tratamiento previo con cirugía endoscópica estándar en pacientes que desarrollaron cicatriz y neosteogénesis en el receso frontal. Además, si bien es controvertido, se decidió realizar un Draf III primario (paciente con antecedente de antrostomías maxilares, pero sin antecedente de cirugía de los recesos frontales previa) debido a la compleja anatomía del receso frontal que presentaba un paciente. Durante la cirugía, considerando la imposibilidad de resecar las celdillas y pólipos de los senos frontales por medio de un abordaje endoscópico estándar, lo que implicaba una alta probabilidad de fracaso quirúrgico y necesidad de un Draf III en un segundo tiempo, se decidió resolver el caso en un solo tiempo realizando un Draf III primario.

En todos los casos de nuestra serie, el abordaje Draf III permitió el adecuado manejo de la patología frontal. En relación a los osteomas frontales de nuestra serie, su localización y extensión frontal permitió su resección endoscópica mediante un Draf III, sin embargo, el abordaje abierto continúa siendo una excelente alternativa para resecar 
osteomas con extenso compromiso de los senos frontales (menor tiempo quirúrgico), especialmente si el osteoma alcanza zonas periféricas de senos frontales con neumatización importante, difíciles o imposibles de alcanzar endoscópicamente. Si bien, muchos mucoceles frontales 0 frontoetmoidales pueden ser drenados con técnica estándar de cirugía endoscópica nasal, creemos que es conveniente estar preparado y consentir al paciente para una eventual conversión a un Draf III. La neosteogénesis que frecuentemente se desarrolla en el receso frontal puede impedir marsupializar adecuadamente el mucocele lo que significará una alta probabilidad de recidiva. En el caso de papilomas invertidos que comprometen extensamente uno 0 ambos senos frontales, creemos que es aconsejable siempre ir preparado para asociar la resección endoscópica con un abordaje abierto ya que muchas veces es difícil predecir con las imágenes la zona y la extensión del sitio de inserción. En nuestra serie, ambos papilomas invertidos presentaban un sitio de implantación al alcance endoscópico mediante un Draf III.

Todos los pacientes de la serie presentaron permeabilidad adecuada del Draf III al último control (8 pacientes amplia y 3 pacientes parcial, pero adecuada). Ningún paciente requirió cirugía de revisión debido a estenosis crítica del Draf III. En relación a este punto, se ha descrito que la estenosis ocurre principalmente en los dos primeros años tras la cirugía ${ }^{6,7}$. Respecto a la permeabilidad de los senos frontales a largo plazo, Naidoo y cols describen los resultados del abordaje Draf III en 229 pacientes operados por RSC con un seguimiento de 45 meses. En esta serie, fueron necesarias cirugías de revisión en $5,2 \%$ de los pacientes. No hubo complicaciones quirúrgicas ni empeoramiento de

\section{BIBLIOGRAFÍA}

1. Draf W. Endonasal micro-endoscopic frontal sinus surgery: The Fulda concept. Oper Tech Otolaryngol Head Neck Surg 1991; 2: 234-40.

2. Scott na, Wormald P, Close D, Gallagher R, Anthony A, Maddern GJ. Endoscopic modified Lothrop procedure for the treatment of chronic los síntomas ${ }^{8}$. Ting y col presentan una serie de 143 pacientes operados de Draf III con un tiempo de seguimiento de 10,2 años. Reportan cirugía de revisión en $26,5 \%$ de los pacientes y una mayor incidencia en casos de tumores y mucocele ${ }^{6}$.

Algunas limitaciones de nuestro estudio son el pequeño tamaño de la población estudiada y el tiempo de seguimiento relativamente corto en un porcentaje significativo de ésta. Estos factores probablemente expliquen la ausencia de revisiones de Draf III por estenosis en nuestra serie. También pueden haber contribuido a este hecho el bajo porcentaje de pacientes con $\mathrm{RSCr}$ recalcitrante con fenotipos más proinflamatorios (ej.: tríada de Samter), Ios cuales tienen la mayor probabilidad de reestenosis luego de un Draf III, y un porcentaje significativo de patología neoplásica en la población estudiada. El abordaje Draf III es seguro y eficiente en manos expertas, y es bien tolerado por Ios pacientes. Sin embargo, es un procedimiento que requiere de un nivel avanzado de entrenamiento en cirugía endoscópica nasal.

\section{CONCLUSIONES}

En nuestra experiencia, el abordaje Draf III fue útil para el manejo de una variada gama de patologías que afectan a los senos frontales incluyendo la $\mathrm{RSCr}$ con pólipos, osteoma, mucocele, papiloma invertido, y para la realización de un abordaje endoscópico extendido a la fosa craneal anterior. La permeabilidad del drenaje frontal obtenida con esta técnica a corto, mediano y largo plazo fue adecuada. Las indicaciones y resultados fueron similares a las series publicadas en la literatura actual.

frontal sinusitis: a systematic review. Otolaryngol Head Neck Surg 2003; 4: 427-38.

3. Shih LC, Patel VS, Choby GW, Nakayama T, Hwang $\mathrm{PH}$. Evolution of the Endoscopic Modified Lothrop Procedure: A Systematic Review and Meta-analysis. Layngoscope 2017; 128: 317-26.

4. Maul X, González C, Callejas C. Osteoma frontoetmoidal, otras aplicaciones del Draf III, 
a raíz de un caso clínico. Rev Otorrinolaringol Cir Cabeza Cuello 2015; 75: 245-50.

5. Muñoz T, Villanueva P, González C, Maul X, Callejas C. Abordaje endoscópico endonasal puro de estesioneuroblastoma. Rev Otorrinolaringol Cir Cabeza Cuello 2017; 77: 57-62.

6. Ting JY, Wu A, Metson R. Frontal sinus drillout (modified Lothrop procedure): long-term results in 204 patients. Laryngoscope 2014; 124: 1066-70.

7. DeConde AS, Smith TL. Outcomes After Frontal Sinus Surgery: An Evidence-Based Review. Otolaryngol Clin North Am 2016; 49: 1019-33.

8. Naidoo Y, Bassiouni A, Keen M, Wormald PJ. Longterm outcomes for the endoscopic modified lothrop/draf III procedure: A 10-year review. Laryngoscope 2014; 124: 43-9. 